\title{
Modeling of a method of parallel hierarchical transformation for fast recognition of dynamic images
}

\author{
Leonid I Timchenko*, Nataliya I Kokryatskaya and Viktoriya V Shpakovych
}

\begin{abstract}
Principles necessary to develop a method and computational facilities for the parallel hierarchical transformation based on high-performance GPUs are discussed in the paper. Mathematic models of the parallel hierarchical (PH) network training for the transformation and a PH network training method for recognition of dynamic images are developed.
\end{abstract}

Keywords: Parallel hierarchical (PH) transformation; $\mathrm{PH}$ network training; Fast recognition of dynamic images; Normalizing equation; Classification and processing of images of extensive laser routes

\section{Introduction}

Rapidly growing requirements to modern computational media encourage the development of new intelligent methods of information transfer and processing. Rigid requirements to real-time information processing systems force scientists to develop and regularly upgrade data transfer systems. Today, most internet channels cannot provide required quality of data exchange between such systems, leading to the congestion of those channels and formation of so-called digital bottlenecks. Large volumes of information may be transferred easily through a fiberoptic cable, but laying such cable is rather expensive, even on short distances. At the same time, this problem can be solve using laser-based technologies [1-3], one of the most promising models of information transfer for the near future. In this case, for instance, tons of full-length films and virtual worlds could be transferred to any part of the globe in a blink of an eye.

Most satellites transmit information, such as TV programs, by means of the microwave radiation, while laserbased information transmission could be hundreds of times faster, considerably increasing a carrying capacity of the channel. The laser-based information transmission requires that both satellite and receiving unit (RU) were positioned

\footnotetext{
* Correspondence: timchen@list.ru

Department of the Telecommunication Technologies and Automation, State Economic Technological University of Transport, 19 Lukashevich Street, Kyiv
} 03049, Ukraine properly. A position of the RU lens, the diameter of which is only several centimeters, must be adjusted to one thousandth degree, or the information will not be transferred.

To operate properly, the system requires that a $\mathrm{RU}$ monitors a satellite at all stages of the system operation. For this purpose, an occurrence of laser beam spot (LBS) on images, namely, their geometrical characteristics distorted by the turbulence and air masses, should be predicted accurately.

However, significant noise distortion of those images can not only considerably worsen forecast results but also prevent the system from responding adequately to variations in the monitored object location. To solve a problem of the efficient forecast, frames of the LBS image sequence should be classified to filter a laser route from images considerably distorted by noise, thus forming a tunnel of reference images [see Additional file 1].

Development of computerized real-time forecasting systems gained new momentum with the emergence of highefficiency automated systems of information collection, processing, and storage. Such systems include a number of tools able to classify and forecast characteristics of respective objects, phenomena, or real-time processes based on special algorithms and geometric characteristics of object boundaries. Fragments of laser beam routes used in the optical communication, navigation, ranging, and military equipment are studied in this paper. The development of the parallel hierarchical $(\mathrm{PH})$ network-based forecasting

\section{首 Springer}


systems allows the solution of a problem in automatic object control, laser-based data transfer, and forecasting the moving optical object behavior.

Today, high-performance fast calculations are extremely important as information volumes are still growing, and computational complexity is increasing. The most significant problems include operative response and real-time decision-making, high-resolution video data processing, and classification and forecasting of quick-changing dynamic data [4-6].

High-performance fast calculations are developed quite well and have many applied aspects, helping solve complex problems. Neurocomputers have many advantages as compared to regular Harvard structure computers, but they also have one significant drawback: their price; as a result, some alternatives should be sought.

One of the most efficient methods to process large data arrays is parallel processing based on specialized system solutions, in particular, on the neural-like parallel hierarchical systems. However, LBS hardware implementation of such systems faces a problem of selecting an adequate platform for fast and efficient parallel processing of data bulk.

A hierarchic transformation is an important and powerful computing operation. This motivates the studies of parallel approaches, such as graphical processing units, or low level complexity algorithms, such as the adaptive resonance theory (ART). Though ART was realized with graphic processing units (GPUs), there are a few hierarchical applications of GPU ART. Every ART layer is propagating in the GPU while being trained at the same time. Experimental results show that for deep trees, graphic processors should be used due to the significant advantage of GPUs.

Programming of GPUs using NVIDIA Compute Unified Device Architecture (CUDA) technology is of interest in the computational systems, especially for population algorithms [7-9]. This is important when GPU [10,11] programming is used to achieve certain advantages in hierarchical transformation algorithms [12,13].

ART is attractive for hierarchical transformation due to its speed and scalability to the parallel transformation [14]. However, GPU-based ART has not been developed earlier. Its only drawback is the rigidity of memory usage for the core value if a set of dynamic arrays is restricted to the CPU exclusively. Typical tree algorithms provide pointers for the creation of both a node and a reference [15], which is inefficient when the CUDA technology is used. In [16-18], the implemented GPU programming focused on the behavior of a single ART unit; however, a hierarchical transformation requires a different approach. Architecture of such transformation is based on the pipeline processing structure [19].

A method of organization of the multi-layer tree structure composed on CUDA platform-based units is described in [20]. Presented experimental results demonstrate an increase in performance for different data sets and parameters that were compared with those obtained with regular CPUs.

A goal of this paper is to develop and apply the suggested method and means of parallel hierarchical transformation for fast recognition of dynamic images, namely, those of LBSs. A method of parallel hierarchical transformation based on the normalizing equation allows computational structures to accelerate. Unlike prevalent types of video information encoding (for instance, differential encoding, code-pulse encoding, and delta modulation), which are based on the sequential encoding of difference information, an approach suggested here processes the results of the data array encoding distributed in the space-time domain.

In addition, the use of the normalizing equation allows a preliminary procedure of image classification by forming tuning coefficients to be realized quite simply, thus determining weighting coefficients for each class. In particular, to perform a real-time classification and analysis of, for instance, adjacent frames of a multimedia image, the use of the normalizing equation gives better results in comparison to other approaches [21].

Specifically, this research aims to analyze a sequence of LBS image frames forming separate routes. Each route is formed from fragments obtained from the receiver with a frequency of $40 \mathrm{~ms}$. Each image of the pattern has a dimension of $128 \times 128$ decomposition elements.

The paper consists of the following parts: Description of parallel-hierarchical transformation algorithms, software modeling and efficiency of the $\mathrm{PH}$ transformation, experimental results and physical modeling of the $\mathrm{PH}$ transformation for fast recognition of LBS images, and conclusions.

\subsection{A method of parallel hierarchical transformation with formation of the normalizing equation for fast image recognition}

Application of the masking method of information processing for fast recognition of LBS images [22] indicates a need to improve a method of the parallel hierarchical transformation. A task to train the suggested PH network by analogy with training in the radial basis function (RBF) networks is in practice brought down to the controlled training of initial-layer network elements. Based on the general idea of the structural organization of artificial neural-like networks as input layer - hidden layer - output layer, it is possible to produce a trainable $\mathrm{PH}$ network, where the first network level serves as an input layer, levels 2 to $k$ (where $k$ is a number of the hidden layer levels) serve as hidden layers, and the output layer, which is traditionally used in artificial neural-like networks, serves as an output layer (Figure 1):

An organizational structure of the multi-layer $\mathrm{PH}$ network is shown in Figure 2.

A number of hidden-layer elements can be determined from the network algorithm length, accordingly formalizing a calculation procedure of the number of hidden-layer 


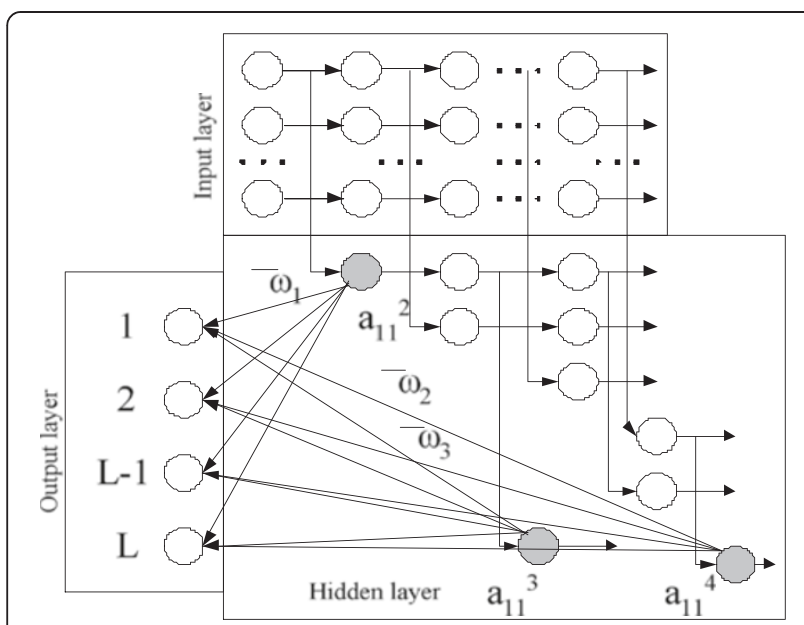

Figure 1 A three-layer structure of the PH network. Grey circles represent the tail elements of the network, and the white circles are their interim elements for various levels.

elements. Averaged values of weighting coefficients are determined by formula (1):

$$
\overline{\mathrm{w}_{\mathrm{t}}^{\mathrm{r}}}=\frac{\sum_{\mathrm{p}=1}^{N} \mathrm{w}_{\mathrm{t}}^{(\mathrm{p})}}{\mathrm{N}}, \mathrm{t}=\overline{1, \mathrm{k}-1}, \mathrm{r}=\overline{1, \mathrm{R}}
$$

where $N$ is a dimensionality of the taught sample $P . r$ is the PH network hidden layer number.

A system of equations to determine tuning coefficients $w_{1}^{r}-w_{k-1}^{r}$ can be composed as system (2):

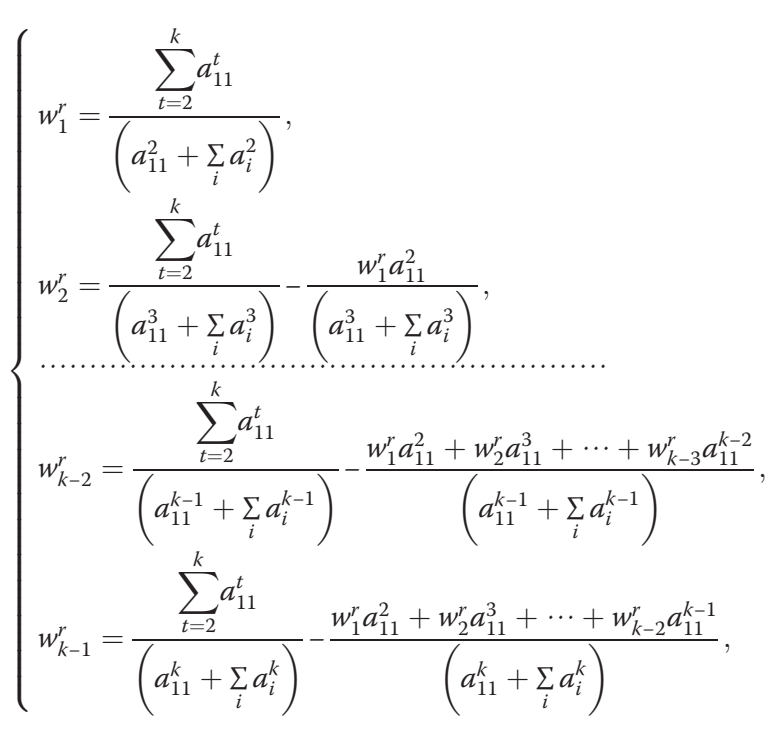

where $\sum_{t=2}^{k} a_{11}^{t}$ is the reference frame component of the extended route being recognized. $\sum_{i} a_{i}^{k}$ and $a_{11}^{2}-a_{11}^{k}$ are

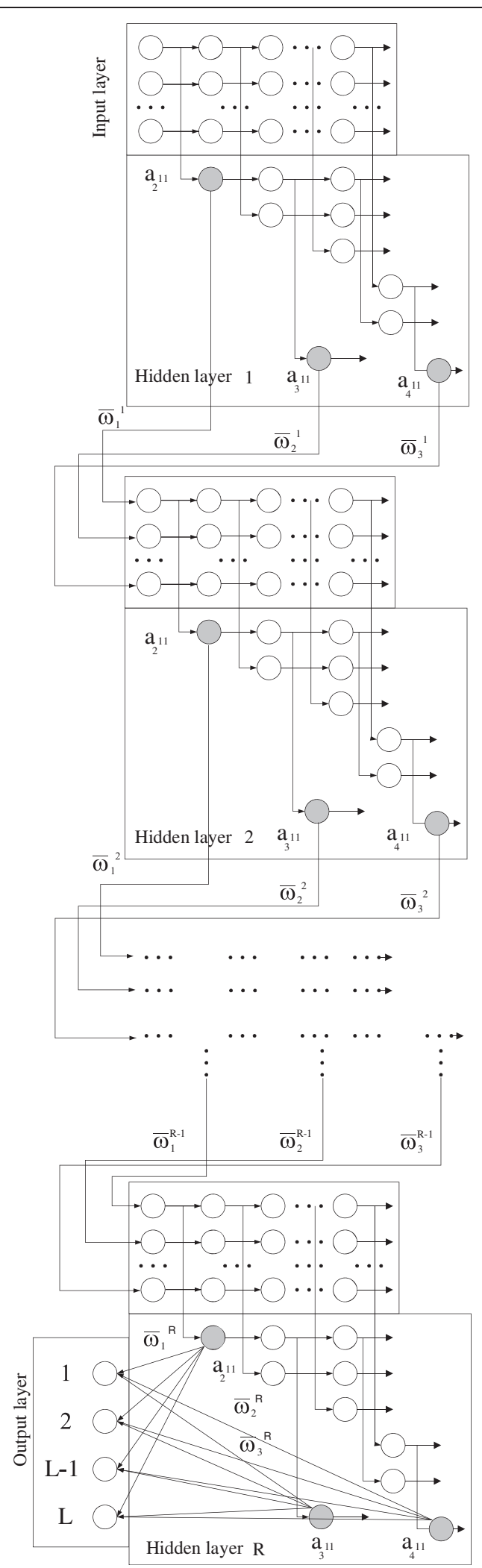

Figure 2 A structure of the multi-layer PH network. Grey circles represent the tail elements of the network, and the white circles are their interim elements for various levels. 
the current frame components of the extended route being recognized.

After finding values $w_{1}-w_{k-1}$, a normalizing Equation (3) can be formed:

$$
d=\frac{\bar{w}_{1}^{R} a_{11}^{2}}{\sum_{t=2}^{k} a_{11}^{t}}+\frac{\bar{w}_{2}^{R} a_{11}^{3}}{\sum_{t=2}^{k} a_{11}^{t}}+\cdots+\frac{\bar{w}_{k-2}^{R} a_{11}^{k-1}}{\sum_{t=2}^{k} a_{11}^{t}}+\frac{\bar{w}_{k-1}^{R} a_{11}^{k}}{\sum_{t=2}^{k} a_{11}^{t}}=\frac{\sum_{t=2}^{k} \bar{w}_{t-1}^{R} a_{11}^{t}}{\sum_{t=2}^{k} a_{11}^{t}}
$$

To normalize results of the $\mathrm{PH}$ network with tuning coefficients (2), the main property of the PH network will be used: $\sum_{t=2}^{k} a_{11}^{t}=\sum_{i} a_{i}$ [21]. Then, a right part of the normalizing Equation (3) represents a ratio of the sum of products of averaged tuning coefficients and tail elements to the sum of the tail elements of the network.

With the correct recognition of LBS images, this normalized measure $d \rightarrow 1$. The similarity of the images being recognized is measured by a degree of its closeness to 1 .

Using the normalizing Equation (3), where $\bar{w}_{1}^{R}=\bar{w}_{2}^{R}$ $=\ldots=\bar{w}_{k-2}^{R}=\bar{w}_{k-1}^{R}=1$, it is possible for a fixed value of $d$ to formulate quite easily a preliminary procedure of image classification and then, according to system (2), form tuning coefficients $\bar{w}_{1}-\bar{w}_{k-1}$, thus determining weighting coefficients for each class.

In particular, for the real-time classification and analysis of adjacent frames of extended laser beam routes, the normalizing Equation (3) acquires form (4):

$$
d=\frac{\left(a_{11}^{2}\right)^{j+1}}{\left(\sum_{t=2}^{k} a_{11}^{t}\right)^{j}}+\frac{\left(a_{11}^{3}\right)^{j+1}}{\left(\sum_{t=2}^{k} a_{11}^{t}\right)^{j}}+\cdots+\frac{\left(a_{11}^{k-1}\right)^{j+1}}{\left(\sum_{t=2}^{k} a_{11}^{t}\right)^{j}}+\frac{\left(a_{11}^{k}\right)^{j+1}}{\left(\sum_{t=2}^{k} a_{11}^{t}\right)^{j}}
$$

where $\left(\sum_{t=2}^{k} a_{11}^{t}\right)^{j}$ is a sum of $k-1$ tail elements. $j$ is a frame number, where $j=1, \bar{m}-1$, and $\left(a_{11}^{2}\right)^{j+1}-\left(a_{11}^{k}\right)^{j+1}$ is a value of tail elements of images of the previous (jth) and following $((j+1)$ th $)$ frames, respectively.

Given the above-mentioned property of the $\mathrm{PH}$ network, the normalizing Equation (3) acquires form (5):

$$
d=\frac{\left(a_{1}\right)^{j+1}}{\left(\sum_{t=2}^{k} a_{11}^{t}\right)^{j}}+\frac{\left(a_{2}\right)^{j+1}}{\left(\sum_{t=2}^{k} a_{11}^{t}\right)^{j}}+\cdots+\frac{\left(a_{N-1}\right)^{j+1}}{\left(\sum_{t=2}^{k} a_{11}^{t}\right)^{j}}+\frac{\left(a_{N}\right)^{j+1}}{\left(\sum_{t=2}^{k} a_{11}^{t}\right)^{j}}
$$

Because in calculating expression (5), there is no need to determine tail elements $\left(a_{11}^{2}\right)^{j+1}-\left(a_{11}^{k}\right)^{j+1}$ to process the $(j+1)$ th frame. A recognition time significantly decreases for normalized results of input data processing $\left(a_{1}-a_{N}\right)$ in comparison to the known neural-like networks, where input data of the $j$ th frame cannot be used for recognition in the initial layer of the $(j+1)$ th frame.

To realize a parallel hierarchical transformation for fast recognition of LBS images, an average value $\bar{a}_{i}^{t}$ should be selected from the available digital information and rounded to the closest integer; then, two connected $\mathrm{PH}$ networks should be formed to process positive and negative difference components. In this case, for each of those PH networks, a system of equations can be formed, where the roots for all equations except for the first one are pairs of tuning coefficients (2):

- for positive difference components: $\left(w_{1}^{r(+)}-w_{k-1}^{r(+)}\right)$

- for negative difference components: $\left(w_{1}^{r(-)}-w_{k-1}^{r(-)}\right)$.

For the first layer of the $\mathrm{PH}$ network, only the tuning coefficient $w_{1}^{r(+)}$ is being calculated because there are only positive difference components here.

When processing a sequence of extended laser route images, it will be more appropriate to use a normalizing equation for two adjacent image frames, where the first image is taken as the reference one. Then, the normalizing equation will have form (6):

$$
\begin{aligned}
d= & \frac{\bar{w}_{1}^{R}\left(a_{11}^{2}\right)^{j}}{\sum_{t=2}^{k}\left(a_{11}^{t}\right)^{j-1}}+\frac{\bar{w}_{2}^{R}\left(a_{11}^{3}\right)^{j}}{\sum_{t=2}^{k}\left(a_{11}^{t}\right)^{j-1}}+\cdots+\frac{\bar{w}_{k-1}^{R}\left(a_{11}^{k-1}\right)^{j}}{\sum_{t=2}^{k}\left(a_{11}^{t}\right)^{j-1}} \\
& +\frac{\bar{w}_{k}^{R}\left(a_{11}^{k}\right)^{j}}{\sum_{t=2}^{k}\left(a_{11}^{t}\right)^{j-1}}=\frac{\sum_{t=1}^{k} \bar{w}_{t}^{R}\left(a_{11}^{t}\right)^{j}}{\sum_{t=2}^{k}\left(a_{11}^{t}\right)^{j-1}}
\end{aligned}
$$

where $\bar{w}_{1}-\bar{w}_{k}$ are tuning coefficients obtained during the preliminary processing on the first images of the set; $\left(a_{11}^{t}\right)^{j}$, $\left(a_{11}^{t}\right)^{j}-1$ are tail elements of the current and previous images, respectively.

Usually, when processing a sequence of extended laser route images, it is necessary to detect 'bad' images, or those internal and external contours of which are most distorted by atmosphere, and exclude those from the route analysis. Because the normable criterion (6) can be found for both a single frame of the laser route, and for the laser route as a whole, we can form a set A from coefficients $d_{i}$ (7):

$$
A=\left(d_{1}, d_{2}, \ldots d_{N}\right)
$$

where $N$ is a number of frames of the laser route.

Because $d$ is a measure of correspondence between tuning coefficients of the reference $\mathrm{PH}$ network and those of the current network, the $i$ th elements of the set 
(7), which have low values, can be classified as 'bad' images:

$$
\left(a_{i, j}^{k(\cdots)}\right)_{e} \bigcap\left(a_{i, j}^{k(\cdots)}\right)_{n}=\left\{\begin{array}{l}
\text { "good"image, if } d_{i} \geq X, \\
\text { "bad"image otherwise }
\end{array}\right.
$$

where $X$ is a determined threshold criterion. As $0 \leq d \leq 1$, then $0 \leq X \leq 1$. Experiment demonstrated that the threshold criterion (9) should be set within limits (9) for insignificant image deformations and within limits (10) in case of more substantial deformations.

For the quality classification and processing of a sequence of extended laser route frames, this threshold criterion may be used in 'tighter' limits (9):

$$
0,8 \leq X \leq 1
$$

For those laser routes which contain images significantly distorted by atmospheric agents, it is recommended to set less strict limits of the threshold value (10):

$$
0,4 \leq X \leq 1
$$

\subsection{The computational efficiency of the developed method of parallel hierarchical transformation in comparison with other existing transformations}

The number of computational operations used for the $\mathrm{PH}$ transformation is $N(N+1)$, where $N$ is the total number of elements processed. For comparison, the number of operations used in other transformations widely applied in practice, e.g., orthogonal transformations, are the following: $4 N^{2} \log _{2} N$ for fast Fourier transformation, $2 N^{2} \log _{2} N^{2}$ for Hadamard, and $4 N(N+1)$ for Haar.

Absence of time-consuming operations indicates a sufficient simplicity of the computational procedure that realizes the parallel hierarchical transformation and makes it an efficient method for use in various applied areas requiring a combination of a high level of the parallelism and a compact form of data representation.

\subsection{Development of the parallel hierarchical transformation algorithms}

A general algorithm for fast recognition of LBS images can be developed based on the suggested method of the parallel hierarchical transformation for processing dynamic images (Figure 3):

This algorithm envisages a call to subroutine NetForm (NETFORM Engineering Machinery Metal Ltd., Turkey), a subprogram serving to construct network structures for images. A detailed description of the subroutine is shown as an algorithm (Figure 4):

This subroutine inputs the initial information to the $\mathrm{PH}$ network. After that, G-transformation of information [21] takes place at all levels of the PH network. The Gtransformation is performed in the following way. A

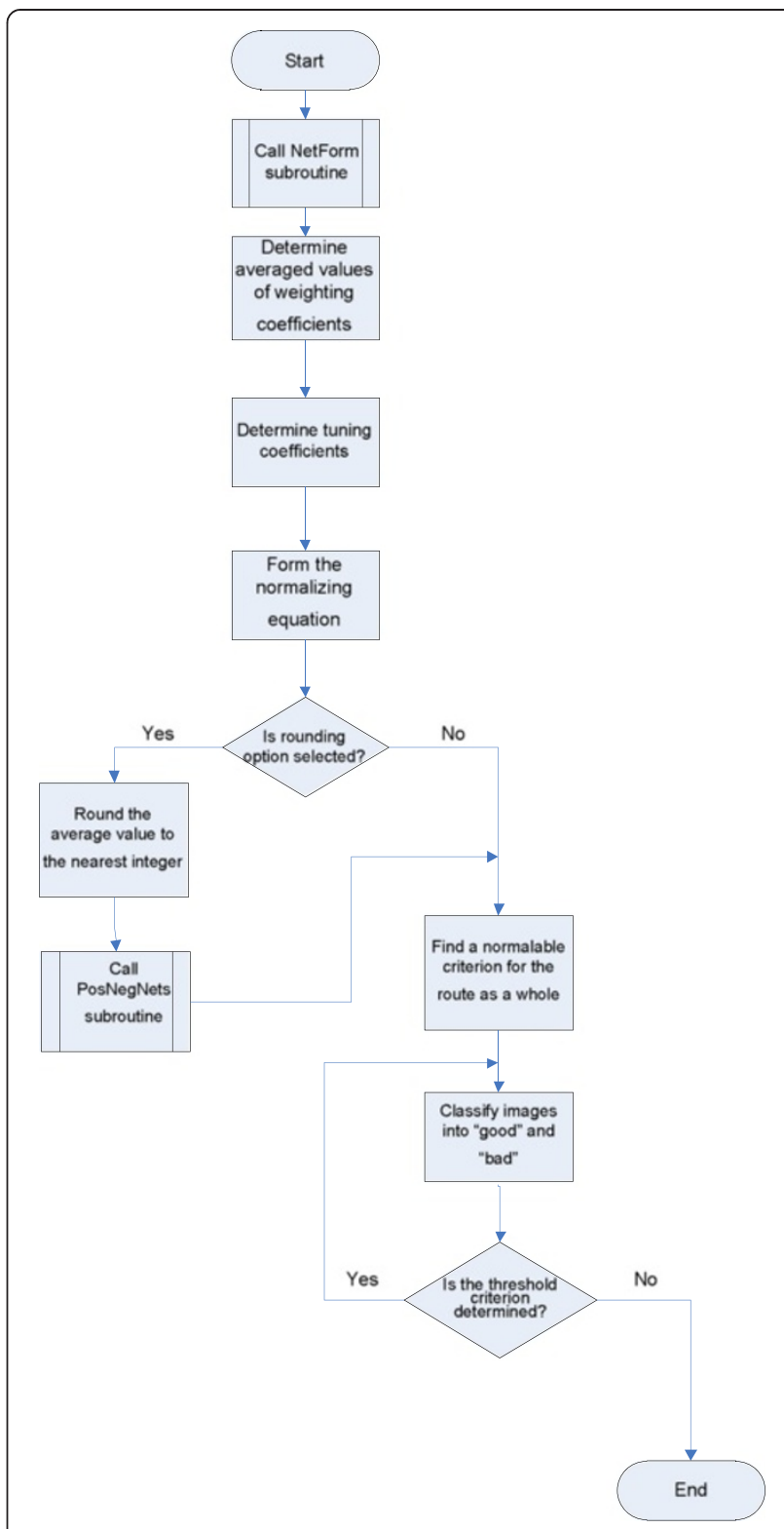

Figure 3 The general algorithm for recognition of images of LBSs.

vector of incoming image is sent to the $\mathrm{PH}$ network input (to input elements of first-level branches of the network, Figure 1). A mathematical model of the parallel decomposition of the set $\mu=\left\{a_{i}\right\}, i=1, n$ [21], used in each branch of the $\mathrm{PH}$ network, looks as follows:

$$
\sum_{i=1}^{n} a_{i}=\sum_{j^{*}=1}^{P}\left(n-\sum_{k=0}^{j^{*}-1} n_{k}\right)\left(a^{j^{*}}-a^{j^{*}-1}\right)
$$

where $a_{i} \neq 0 . P$ is a dimensionality of this set (a number of set elements) or a dimensionality of the $\mathrm{PH}$ network branch of its every level. $a^{z}, z=\overline{1}$, and $\bar{P}$ are the 


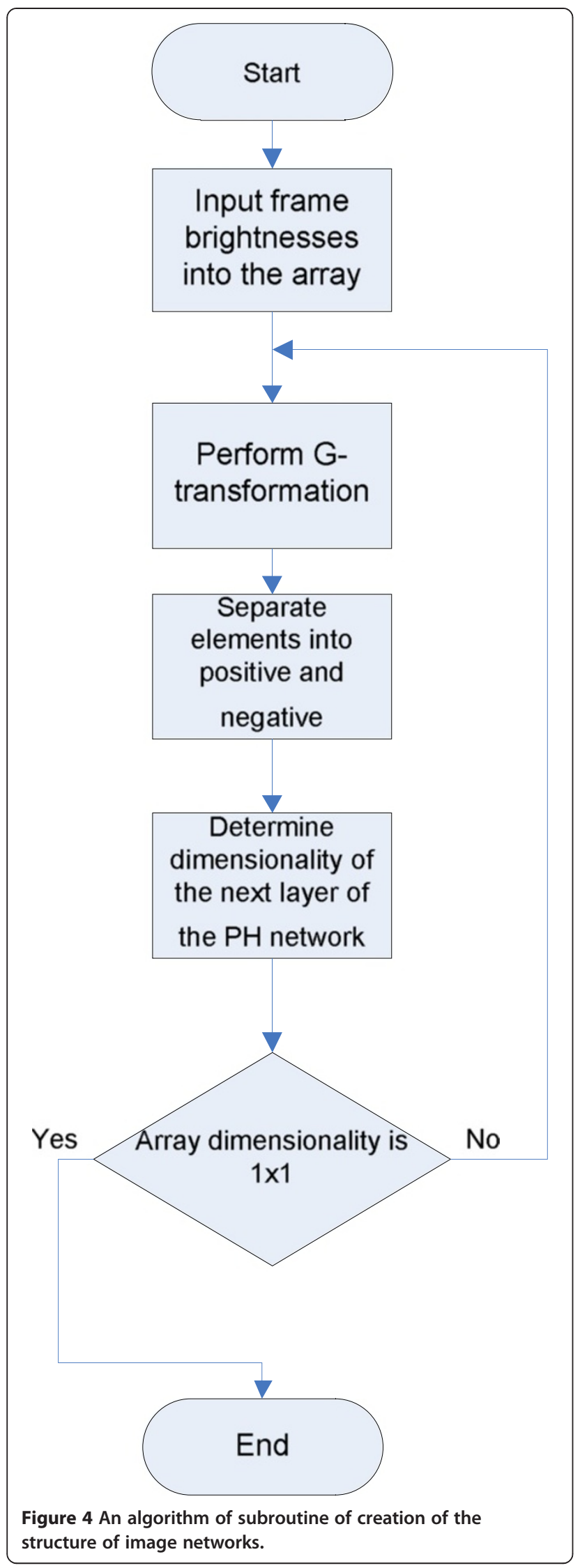

elements of subsets consisting of equal elements; $n_{z}$ is a number of elements in the $k$ th subset (i.e., order of number $\left.a_{i}\right)$, and $a^{i^{*}}$ is an element of the set $\left\{a^{z}\right\}$, selected at the $j^{*}$ th step, $j^{*}=1, P, a^{0}=0, n_{0}=0$.

When a structure of the two networks is constructed, the average values of weighting coefficients and tuning coefficients (4) are being determined. Based on the calculated tuning coefficients, a normalizing Equation (6) is being formed.

If an average value $\bar{a}_{i}^{t}$ is rounded to the closest integer, subroutine PosNegNets is called. It is a subprogram that forms two connected PH networks for the processing of positive and negative difference components. A formation algorithm of the two networks is similar to the

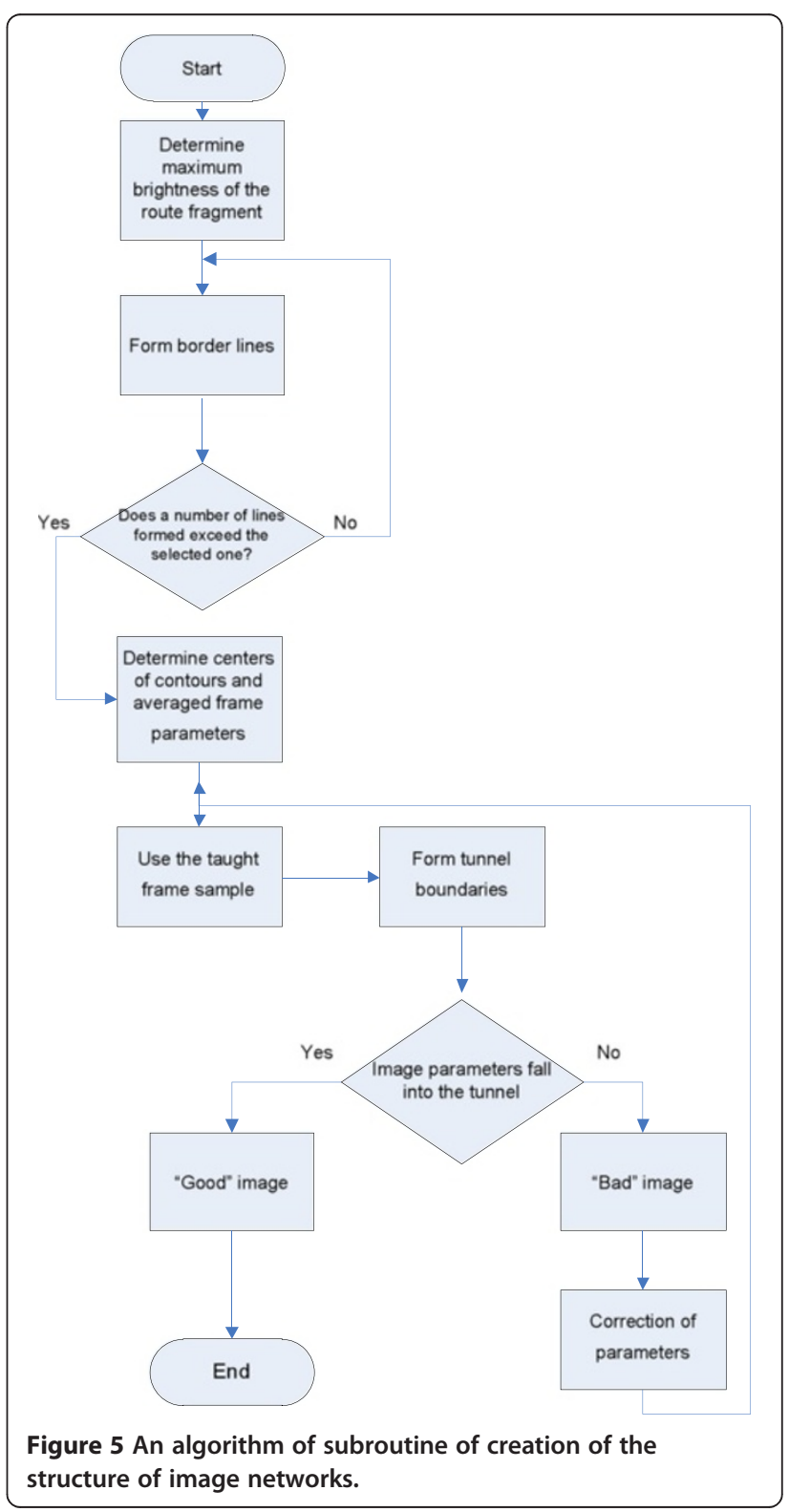



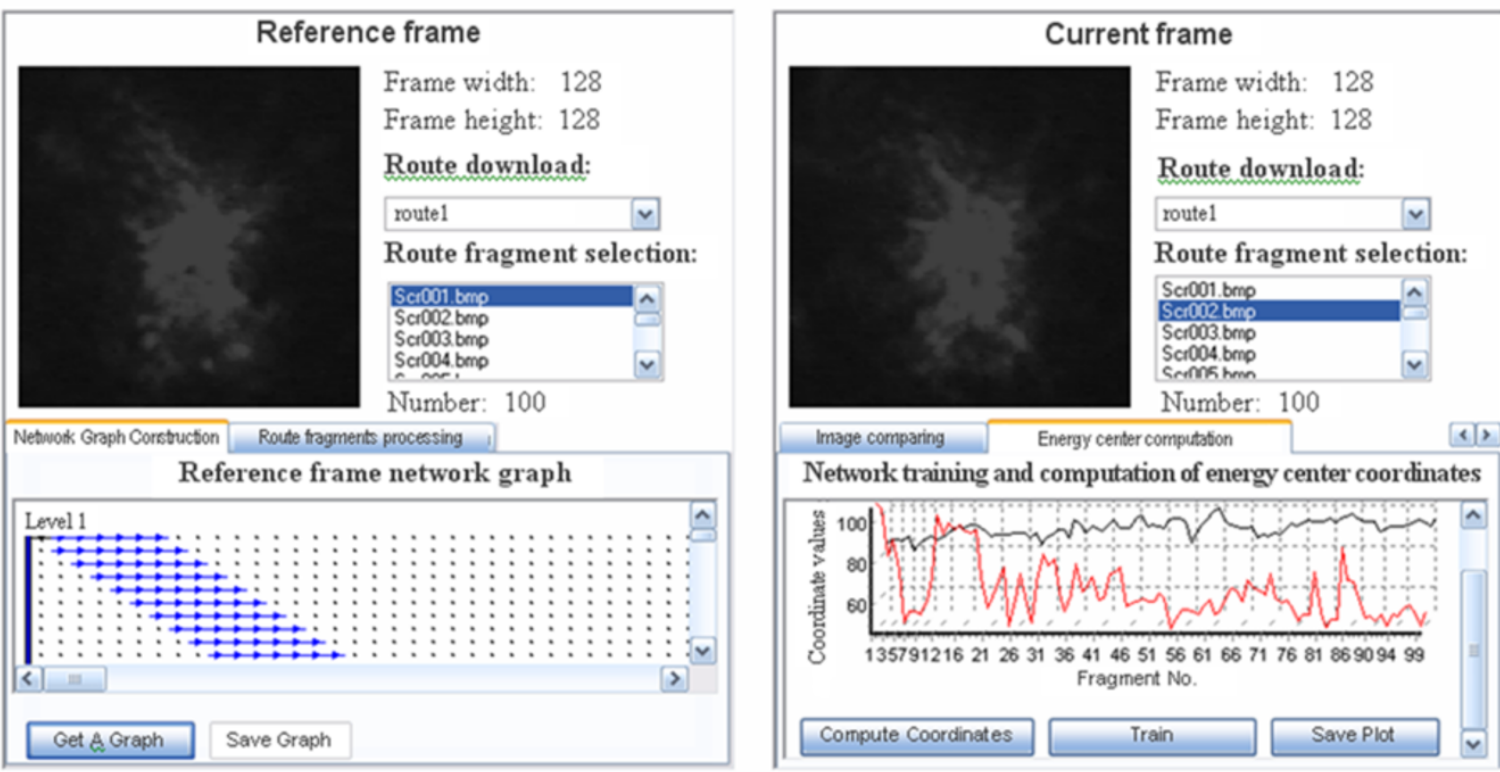

Figure 6 Screen form of the PH network application for fast recognition of laser beam spot images.

formation algorithms of the network structure for images. After that, a normalizing criterion (7) for the route as a whole is being found with further classification of images into good and bad according to ratio (8). If the threshold criterion is determined, the classification is conducted again. Let us develop a preliminary processing algorithm of route fragments (Figure 5).

An image of the laser route fragment arriving to the system input undergoes the following preliminary processing:
1. Determine maximum fragment brightness to form $n$ border lines.

2. Having selected a necessary number of boundaries, find their geometrical centers and average parameters for the whole frame.

3. Form tunnel boundaries using a trained sample and averaged frame parameters.

4. Check if calculated frame parameters are included into the tunnel. Based on this verification, frame image classification is performed. If an image was

Table 1 Comparative characteristics of means of the parallel hierarchical transformation

\begin{tabular}{|c|c|c|c|c|c|}
\hline \multirow[t]{2}{*}{ Number } & \multirow[t]{2}{*}{ Parameter } & \multicolumn{4}{|c|}{ Indices } \\
\hline & & $\begin{array}{l}\text { Known soft hardware } \\
\text { (imitational modeling } \\
\text { based on MLP and } \\
\text { RBF networks [23]) }\end{array}$ & $\begin{array}{l}\text { Known soft hardware } \\
\text { (imitational modeling } \\
\text { based on neural-like } \\
\text { network technology } \\
{[22] \text { ) }}\end{array}$ & $\begin{array}{l}\text { Known soft hardware } \\
\text { (central processor, } \\
\text { PH transformation } \\
\text { method based on the } \\
\text { Q-decomposition [19]) }\end{array}$ & $\begin{array}{l}\text { Suggested soft hardware } \\
\text { (graphical processor, } \\
\text { method of the PH network } \\
\text { training based on the } \\
\text { normalizing equation) }\end{array}$ \\
\hline 1 & $\begin{array}{l}\text { Average size of good route } \\
\text { fragments (\%) }\end{array}$ & 70 & 74 & 50 & 18 \\
\hline 2 & $\begin{array}{l}\text { Average value of correct } \\
\text { recognition (\%) }\end{array}$ & 92 & 92,5 & 84,8 & 94 \\
\hline 3 & $\begin{array}{l}\text { Accuracy of the energy center } \\
\text { determination, decomposition } \\
\text { elements }\end{array}$ & 1.5 & 1.5 & 1.2 & 0.01 \\
\hline 4 & $\begin{array}{l}\text { Average recognition time of } \\
\text { network processing(s) }\end{array}$ & - & - & 8.4 & 1.52 \\
\hline 5 & $\begin{array}{l}\text { Average time of preliminary } \\
\text { processing of route } \\
\text { fragments(s) }\end{array}$ & - & - & 3.32 & 0.6 \\
\hline
\end{tabular}




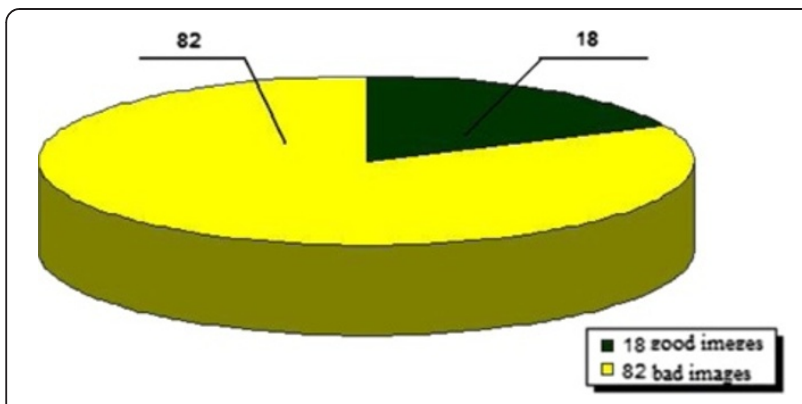

Figure 7 Determination of the normalized measure for further classification of route 1 fragments.

classified as bad, the image parameters are corrected and included into the taught sample; then, an additional training of the system is performed.

\subsection{Program modeling and application efficiency of the} parallel hierarchical transformation for fast recognition of LBS images

The developed application is intended to process and classify the LBS images. The software window (Figure 6) represents a form divided into two parts: the left part contains a processing panel of the reference frame of the LBS images route, and the right one that of the current frame of the LBS images route.

Information about the frame parameters is exhibited in the upper part:

- frame width, pixels;

- frame height, pixels;

- information about the route containing the frame;

- frame file name;

- number of frames in the route.

The bottom part contains tabs:

- construction of the network graph, which can be obtained and saved as a PH network graph file;

- route fragment processing, where a route fragment can be processed;
- image comparison, where LBS images being compared and classified;

- determination of energy centers, where LBS energy centers are determined and the PH network is trained.

An energy center is a point in the image, where coordinates $(x, y)$ of a two-dimensional signal with a specific nonlinear density $w(f(x, y))$ meet a given value of $f(x, y)$ and are expressed as follows:

$$
\begin{aligned}
& x_{\mathrm{ec}}=\frac{1}{M} \sum_{x=0}^{N-1} \sum_{y=0}^{N-1} w(f(x, y)) \cdot x \\
& y_{\mathrm{ec}}=\frac{1}{M} \sum_{x=0}^{N-1} \sum_{y=0}^{N-1} w(f(x, y)) \cdot y \\
& M=\sum_{x=0}^{N-1} \sum_{y=0}^{N-1} w(f(x, y)),
\end{aligned}
$$

where $M$ is the first order moment of inertia.

To estimate the efficiency of the suggested algorithms and the soft hardware that developed on their basis, let us describe the technical advantages of means of the $\mathrm{PH}$ transformation as demonstrated by the developed products and conducted experiments. The comparative characteristic is shown in Table 1.

The use of neural-like network technologies for the classification of extended laser route images is analyzed in [22]. The computer modeling revealed $92.5 \%$ correctly recognized images, including $74 \%$ good images and $60 \%$ bad images. In [23], a recognition system was modeled on the basis of the MLP neural-like network and RBF-based neural-like network. Recognition of a sample that included 140 laser route spot objects by the RBF-based neural-like network modeled in the Statistica Neural Networks 4.0 (StatSoft Inc., Moscow, Russia) yielded 92\% correctly

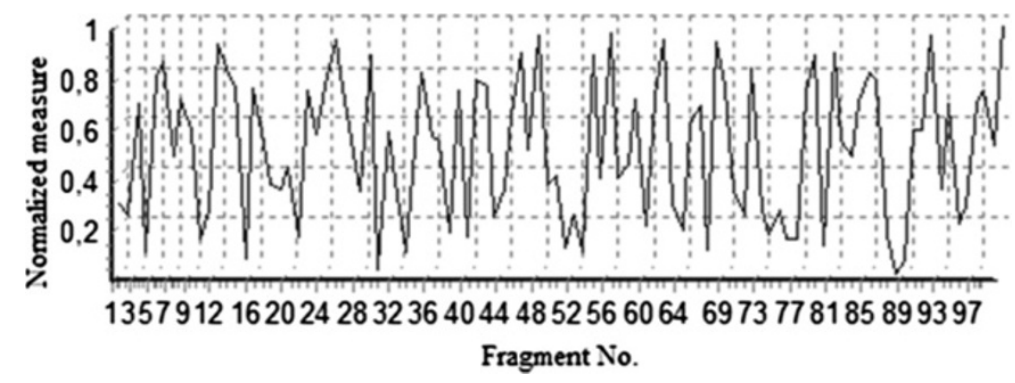

Figure 8 Classification of route 1 fragments (with the threshold criterion (8) $0,8 \leq X \leq 1$ ). 


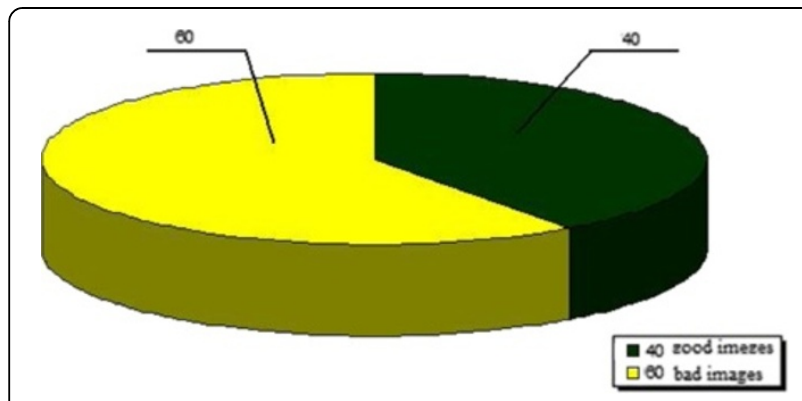

Figure 9 Classification of route 1 fragments (with the threshold criterion (8) $0,6 \leq X \leq 1)$.

recognized images. Comparing the results of $[22,23]$ and those studied in this article, the latter has more potential.

Therefore, the suggested method, algorithms, and soft hardware permit to measure the energy center coordinates of the laser route fragment images based on the normalizing equation with accuracy of no more than 0.01 decomposition elements, thus exceeding known methods (e.g., based on determination of the gravity center using the method of moment characteristics [24-27]) by accuracy 1.5 times on average, which is reflected in Table 1 . A time necessary for preliminary and network processing of laser route fragment images decreases, too.

\section{Experimental results and physical modeling of the parallel hierarchical transformation for fast recognition of LBS images}

Four routes, each containing 100 frames, were used in the experimental research of the parallel hierarchical transformation for fast recognition of LBS images. Using the normalizing Equation (6), let us determine the normalized measures for route 1 (Figure 7).

Using Equations (8) and (9), let us classify the images (Figure 8).

As we see, a portion of good images is $18 \%$. As a rule, in real conditions, positions of energy centers cannot be determined absolutely accurately; therefore, less tight limits

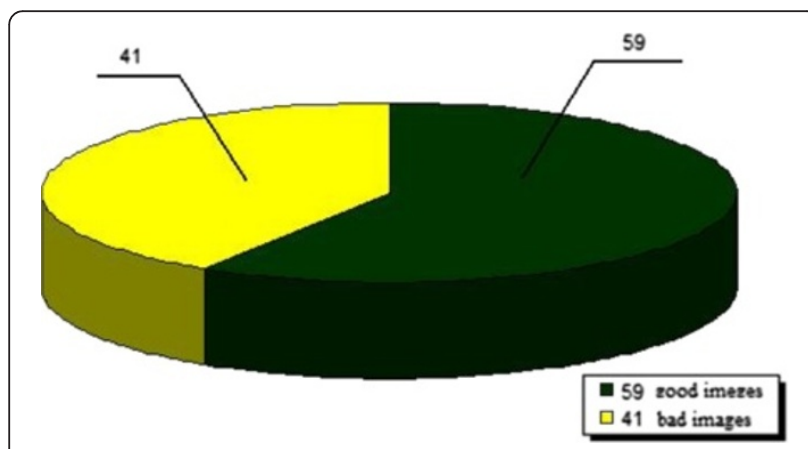

Figure 10 Classification of route 1 fragments (with the threshold criterion (8) $0,4 \leq X \leq 1$ ). a)

\begin{tabular}{|c|c|c|c|c|c|c|c|c|c|c|c|}
\hline 47 & 2 & 3 & 57 & 5 & 12 & 7 & 8 & & & & \\
\hline 10 & 20 & 6 & 13 & 14 & 15 & 16 & 17 & & & & \\
\hline 19 & 11 & 21 & 22 & 23 & 68 & 25 & 26 & & & & \\
\hline 38 & 29 & 64 & 31 & 32 & 33 & 35 & 34 & & & & \\
\hline 37 & 28 & 39 & 49 & 53 & 42 & 41 & 52 & 47 & 57 & 15 & 17 \\
\hline 46 & 1 & 48 & 40 & 61 & 51 & 44 & 43 & 38 & 64 & 68 & 35 \\
\hline 55 & 71 & 4 & 58 & 69 & 62 & 50 & 60 & 46 & 49 & 61 & 52 \\
\hline 30 & 65 & 66 & 67 & 24 & 59 & 70 & 56 & 71 & 67 & 69 & 70 \\
\hline
\end{tabular}

b)

\begin{tabular}{|c|c|c|c|c|c|c|c|c|c|c|c|}
\hline 47 & 2 & 3 & 57 & 5 & 12 & 7 & 8 & & & & \\
\hline 10 & 20 & 6 & 13 & 14 & 15 & 16 & 17 & & & & \\
\hline 19 & 11 & 21 & 22 & 23 & 68 & 25 & 26 & & & & \\
\hline 38 & 29 & 64 & 31 & 32 & 33 & 35 & 34 & & & & \\
\hline 37 & 28 & 39 & 49 & 53 & 42 & 41 & 52 & 47 & 57 & 15 & 17 \\
\hline 46 & 1 & 48 & 40 & 61 & 51 & 44 & 43 & 38 & 64 & 68 & 35 \\
\hline 55 & 71 & 4 & 58 & 69 & 62 & 50 & 60 & 46 & 49 & 61 & 52 \\
\hline 30 & 65 & 66 & 67 & 24 & 59 & 70 & 56 & 71 & 67 & 69 & 70 \\
\hline
\end{tabular}

Figure $11 \mathrm{~A}$ diagram of pixel processing using the Radeon X1300 graphic adapter.

should be set for the threshold criterion using relation (10) (Figures 9 and 10):

An average portion of the good images in this case is $40 \%$ and $59 \%$, respectively. Then, the PH network is trained for repeated processing of the bad route fragments. In physical modeling of the method of $\mathrm{PH}$ transformation and determination of extended laser rout image coordinates using the graphic adapter Radeon X1300 (Advanced Micro Devices, Inc., Sunnyvale, USA), processing of a single image takes about $0.6 \mathrm{~s}$. Radeon X1300 is a basic solution in the ATI Radeon family of graphic adapters based on the graphic processor RV515 with main characteristics of four pixel pipelines, four texture units, and two vertex pipelines [28].

An approach of flow programming is suggested to program the GPU. This approach includes splitting a program into relatively small stages (core programs) that process data flow elements. Core programs go into shaders, and data flows go into textures in the GPU. A shader is a program performed on the GPU and processes a data flow. The 'data flow' means information about coordinates of polygon vertices, each vertex illumination, normal, texture coordinates, and so on, which 


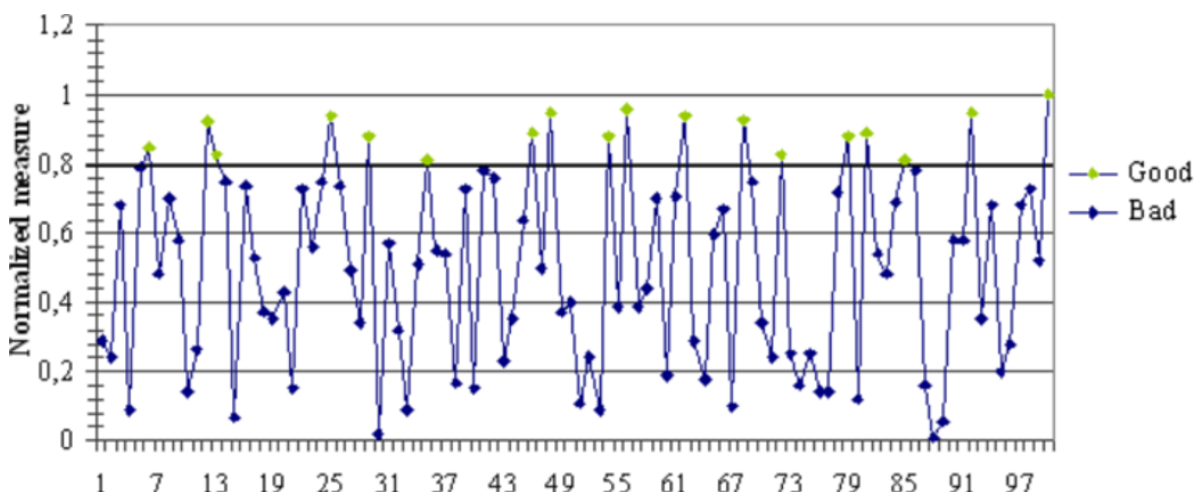

Figure 12 A diagram of formation of good and bad images in route 1.

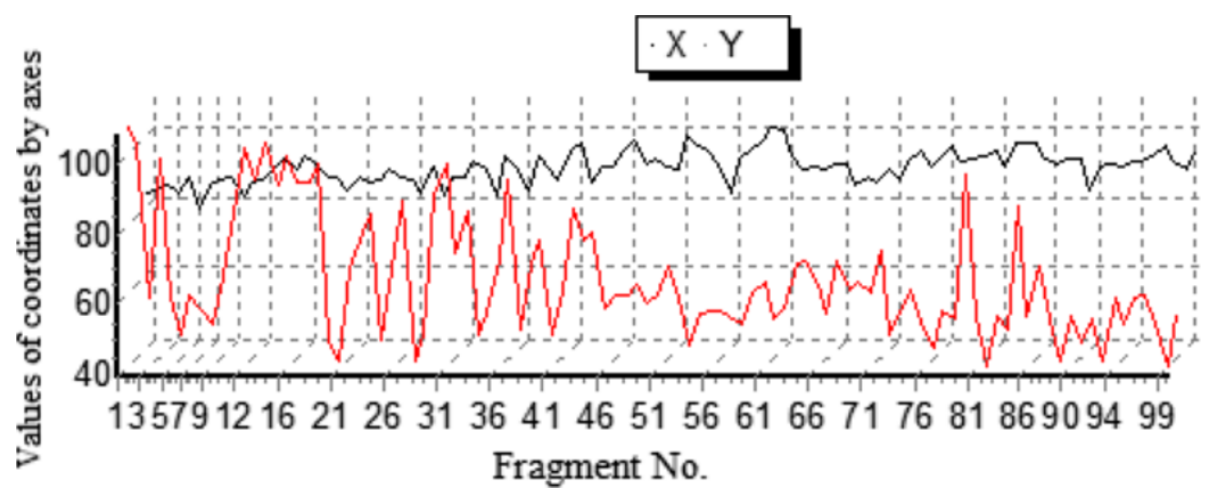

Figure 13 Determination of coordinates of energy centers of Route 1 fragments.

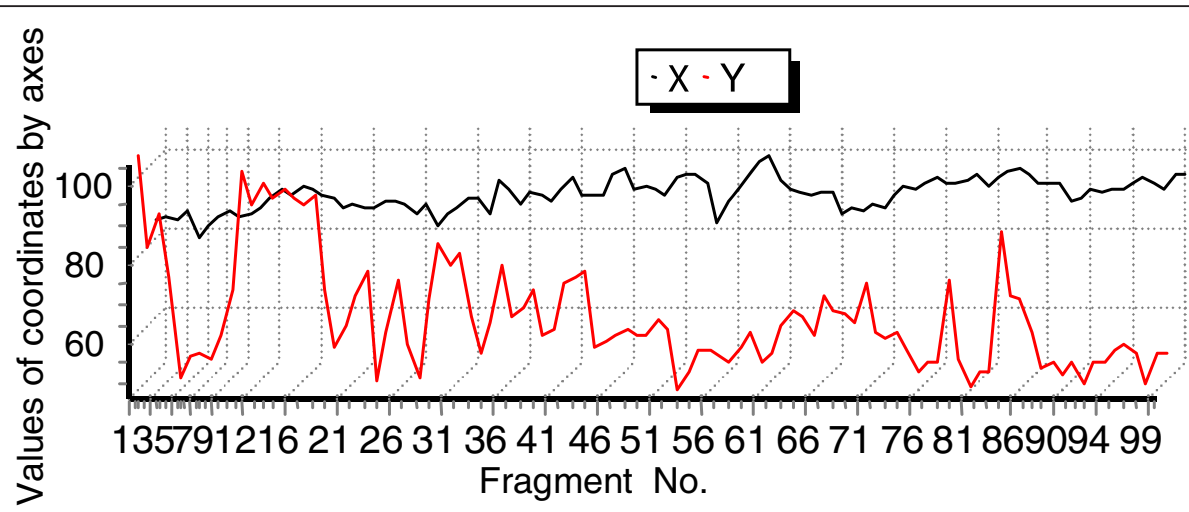

Figure 14 Determination of coordinates of energy centers of route 1 fragments after training of the PH network. 


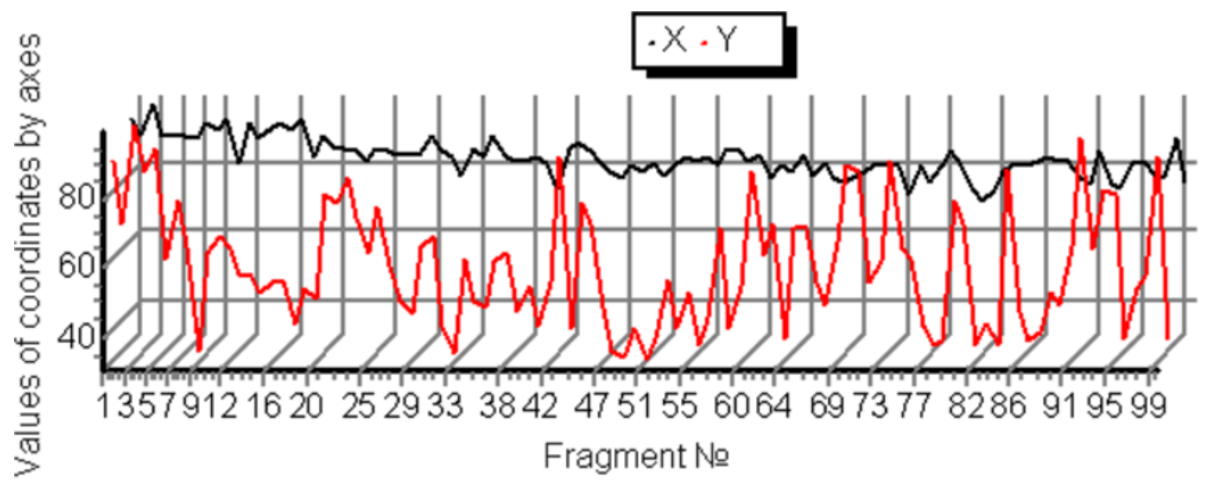

Figure 15 Determination of energy center coordinates of the route 2 fragments.

permanently arrives in blocs to the shader program. A video processor receives outgoing information for all three vertices of the polygon. Having data on the three vertices, the GPU goes through all pixels of a single polygon, while gradually changing (interpolating) vertex values from one vertex to another. A shader length is a memory space of the shader program. Considering that data arriving to the shader is random for the programmer in a sense that an order in which the data will be sent to the shader is not preset, the shader program does not have any statistical or general variables. Otherwise, the shader program is similar to a regular $\mathrm{C}$ program. There are input, output and time variables, standard functions, structures, arrays, cycles, and conditional operators.

Image processing takes place on the GPU with cores realized at all elements covered by the initial region. The only obvious way to calculate a scalar of the input vector is to represent $1 \times 1$ initial elements and use a core that is read in all values from the input texture. This approach has several drawbacks. First, only one of the parallel elementary processors would be busy. Second, that would probably exceed a maximum memory size, including memory of the shader and static instruction of calculation for some hardware. That is why we will perform a parallel operation of reduction based on global methods of communication on parallel computers.

At the high level, GPU-based parallel calculation is a correction of sizes of the input and output texture and of index elements. For the presented vector $M$ of the length $M$, the output of the first step is $M / 2$ with texture $M / 2$. For each of its elements, the coordinates for input texture are corrected in such a way that they correspond to disconnected $2 \times 2$ subregions. Then, the values in those subregions are compared. This is repeated recursively until the $2 \times 2$ texture is reduced finally to the $1 \times 1$ 'scalar' texture through a logarithmic series of repetitions.

Next, the series of images finalize the first step of the $8 \times 8$ algorithm reduction of the input texture (Figure 11a).

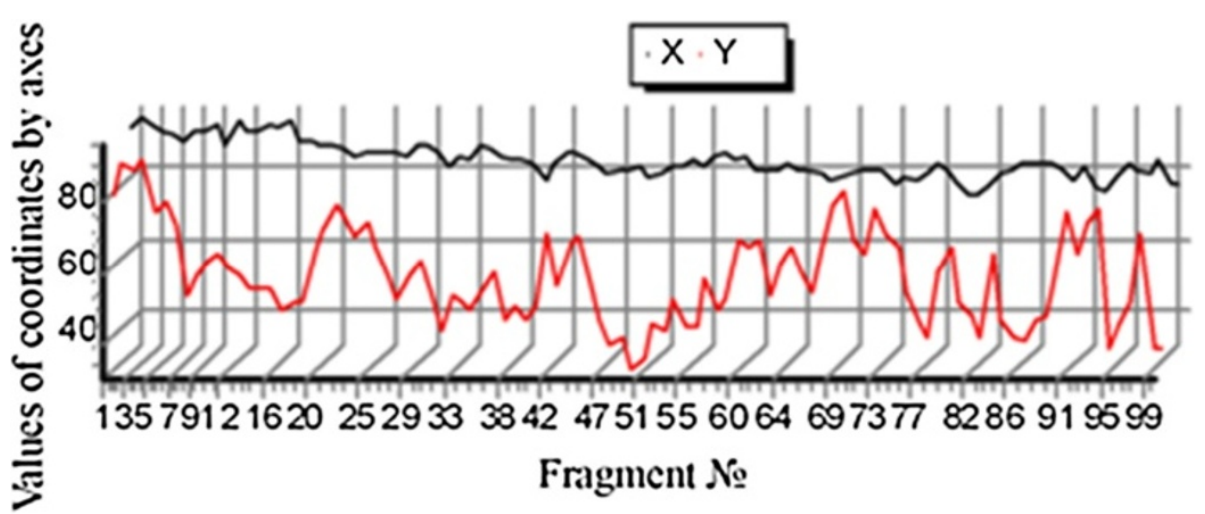

Figure 16 Determination of energy center coordinates of route 2 fragments after the PH network training. 


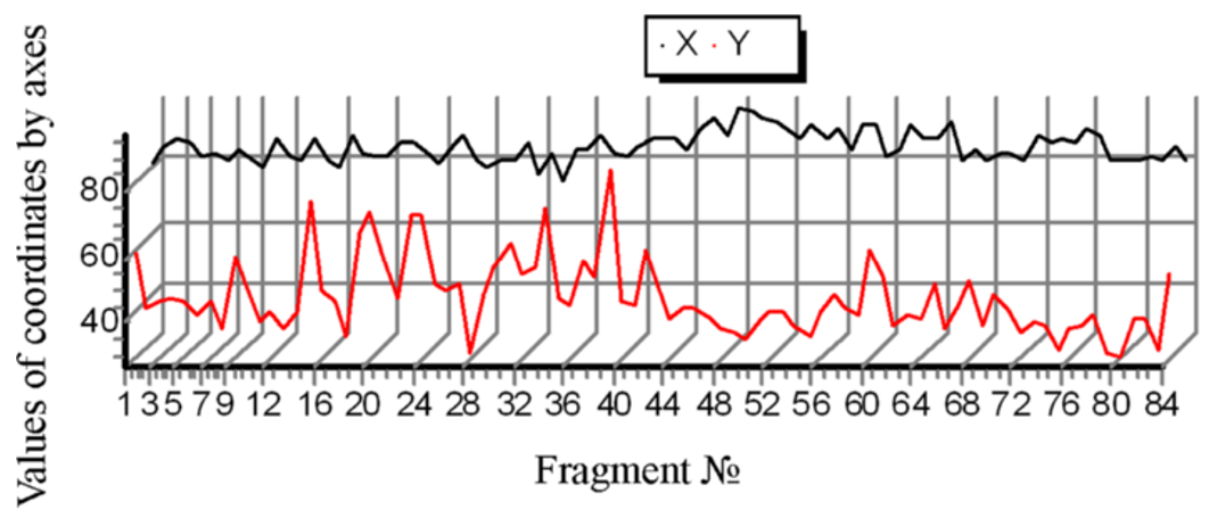

Figure 17 Determination of energy center coordinates of route 3 fragments.

Figure 11a demonstrates the input texture. The initial elements are marked in green. Figure $11 \mathrm{~b}$ is a result of the first round of reduction. Each initial element contains a local transfer maximum of the $2 \times 2$ subregion in the input texture (marked in blue and orange). This relation, in addition, is distinguished in the second line of images (Figure 11b).

Let us determine the coordinates of the energy centers of the fragment of route 1 (Figure 12).

A diagram of changes in the energy center coordinates of route 1 fragments is shown in Figure 13.

After the PH network training, a portion of the good images was $83 \%$ (as compared to $18 \%$ ). The graphic interpretation of the determination of energy center coordinates after the $\mathrm{PH}$ network training is demonstrated in Figures 14 and 15.

After the $\mathrm{PH}$ network training, a portion of the good images of route 2 was $76 \%$ ( $12 \%$ before training), as shown in Figures 16 and 17.
After the $\mathrm{PH}$ network training, a portion of the good images of route 3 fragments was $65 \%$ (15\% before training), as shown in Figures 18 and 19.

After the $\mathrm{PH}$ network training, a portion of good images of route 3 reached $83 \%$ as compared to $17 \%$ before training (Figure 20).

\section{Conclusions}

The paper deals with an important problem of improved efficiency of dynamic image recognition. The analyses of modern trends in the development of fast recognition technologies for dynamic images, as well as the analysis of the $\mathrm{PH}$ transformation development, classify the suggested parallel hierarchical approach as a neural-like method of transformation with a network of direct propagation and the space-time organization of connections. Authors think that the efficiency of computational structures can be increased by applying a developed method of $\mathrm{PH}$ transformation with the formation of the normalizing equation.

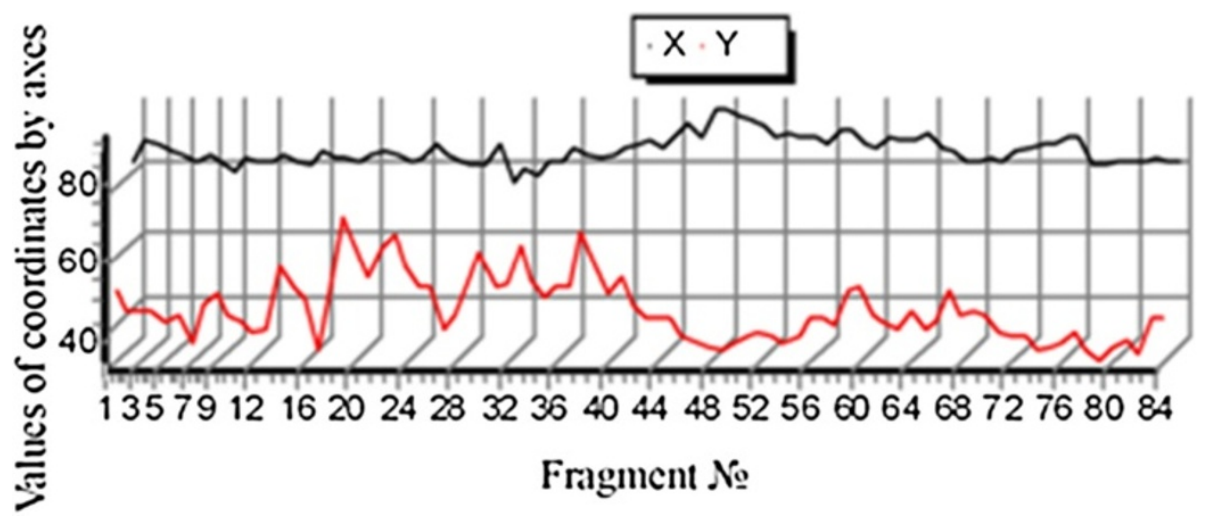

Figure 18 Determination of energy center coordinates of the route 3 fragments after the PH network training. 


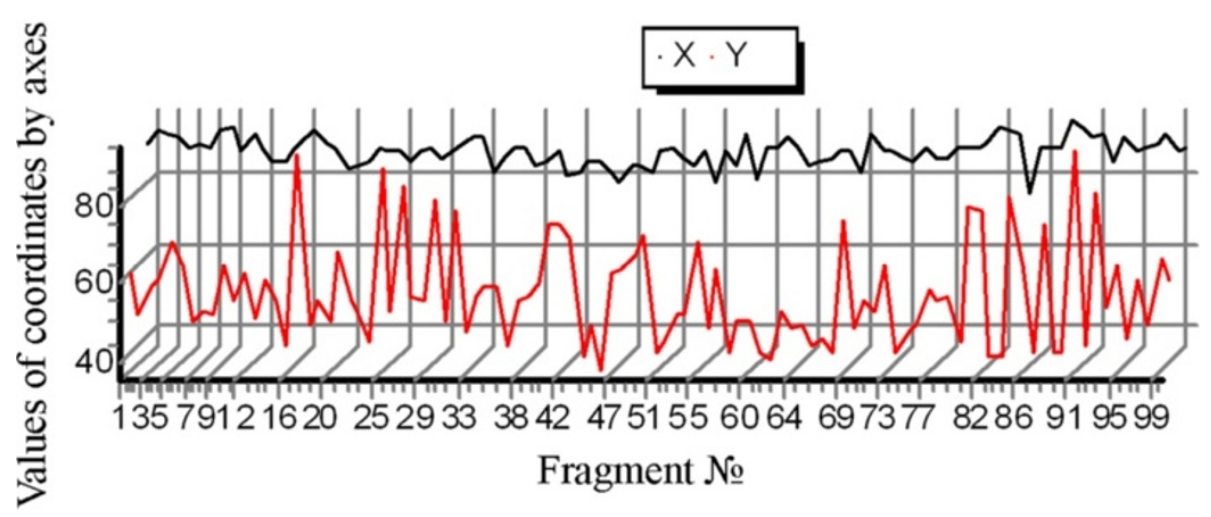

Figure 19 Determination of energy center coordinates of the route 4 fragments.

For quality classification and processing of a sequence of extended laser route spot images, a threshold criterion was introduced. Methods of $\mathrm{PH}$ transformation were developed by introducing a set A that contains measures of correspondence between the reference and current $\mathrm{PH}$ network tuning coefficients and by finding a measure of correspondence between two networks as a whole.

Using a normalizing equation, the processing and recognition algorithms for LBS images were developed, and the $\mathrm{PH}$ network structures for images based on G-transformation were constructed. The developed algorithms allow the determination of energy center positions and classify laser route image frames. Peculiarities of real-time realization of the algorithm of the developed method for route fragments are discussed, and a choice of means for software development is explained. On the basis of the developed algorithms, software was created for modeling a neural-like parallel hierarchical network, which was used for image classification and fast processing. Experimental methods to study the processing of LBS image fragments were developed, which helped in verifying the adequacy of the developed algorithms and in obtaining the results of fast processing and classification of LBS image fragments.

In the conducted experiments, an average portion of good route fragments was $17 \%$, as compared to $50 \%$ in [21]. However, after the training, it increased to $79.25 \%$ (as compared to 65\% [21]). It was also established that an average rate of correct recognition was $94 \%(84.8 \%$ [21]), and the accuracy of energy center determination was no more than 0.01 decomposition elements (as compared to 1.2 decomposition elements [20]), which is reflected in Table 1. This is, on average, 1.5 times higher than the accuracy of known methods, for instance, based on the gravity center determination using a method of moment characteristics. Physical modeling of the method of $\mathrm{PH}$ transformation for fast image recognition using the Radeon X1300 graphic adapter demonstrates that processing of one image requires approximately $0.6 \mathrm{~s}$ (3.32 s [21]), and the network processing requires $1.52 \mathrm{~s}(8.4 \mathrm{~s}[21])$.

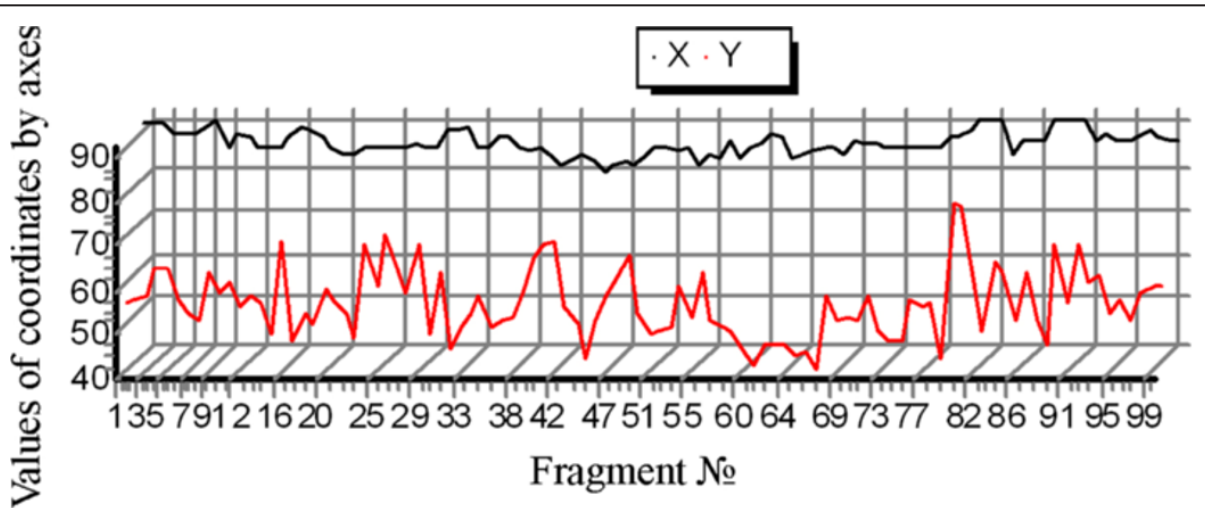

Figure 20 Determination of energy center coordinates of the route 4 fragments after the PH network training. 


\section{Additional file}

Additional file 1: Title: Importance of laser beam profiling systems in telecommunications. Description: Importance of research in the area of development of new methods and means of laser beam profiling and classification of laser spot images in telecommunication systems is explained in this video. A need to use developed means in the tasks of image transfer, processing, and classification for optical communications is demonstrated.

\section{Competing interests}

The authors declare that they have no competing interests.

\section{Acknowledgments}

The authors express their gratitude to the scientific co-director of the work Yuri Kutaev for problem formulation and for his participation in its practical realization. Authors express their sheer gratitude to the team of the Chair of the Telecommunication Technologies and Automatics of the State Economy and Technology University of Transport (Kyiv, Ukraine) for the valuable comments and recommendations expressed in the course of the experimental research. Authors also thank Rector M.V. Makarenko, Vice Rector on Scientific Work Associate PhD V.I. Danilevskiy, and Dean of the Department of Infrastructure and Rolling Stock of Railway Transport Professor A.D. Voznenko for the provided facilities and equipment for scientific studies of this problem in the framework of the state-financed scientific research topics.

Received: 14 August 2012 Accepted: 31 March 2013

Published: 25 April 2013

\section{References}

1. JM Khosrofian, BA Garetz, Measurement of a Gaussian laser beam diameter through the direct inversion of knife-edge data. J Appl Opt 22, 3406-3410 (1983)

2. NG Basov, EM Zemskov, RI II'kaev, VM Kazansky, GA Kirillov, SM Kulikov, YF Kutaev, OY Nosach, SA Sukharev, Laser system for observation and removal of space debris, in Proceedings of GCL/HPL 98, vol. 3574, eds. A Boreisho and GA Baranov (SPIE, Bellingham, 1998), pp. 219-228

3. LI Timchenko, YF Kutaev, VP Kozhemyako, Method for training of a parallelhierarchical network, based on population coding for processing of extended laser paths images, in SPIE Proceedings, ed (Bellingham, AG Tescher (SPIE, 2002). pp. 465-479

4. GPGPU, General purpose computations on graphic processing unit. http://www.gpgpu.org. Accessed 16 April 2013

5. Wikipedia, Open computing language. http://en.wikipedia.org/wiki/OpenCL. Accessed 16 April 2013

6. NVIDIA Corporation, NVIDIA homepage. (2013). http://www.nvidia.ru/page/ home.html. Accessed 16 April 2013

7. DM Chitty, A data parallel approach to genetic programming using programmable graphics hardware, GECCO '07, in Proceedings of the 9th annual conference on genetic and evolutionary computation, ed. by JF Miller (ACM, New York, 1991). vol. 2, pp. 1566-1573

8. Z Luo, H Liu, X Wu, Artificial neural network computation on graphic process unit, IJCNN '05. Paper presented at the IEEE international joint conference on neural networks, Florida. 31 July-4 Aug 2005

9. JM Li, DL Wan, ZX Chi, XP Hu, A parallel particle swarm optimization algorithm based on fine-grained model with GPU accelerating. Harbin Gongye Daxue Xuebao/J Harbin Inst Technol 38(12), 2162-2166 (2006)

10. C Chun-Yuan, C Chih-Hao, C Min-An, W An-Yeu, EURASIP Multi-prediction particle filter for efficient parallelized implementation. J Adv Signal Process 2011, 53 (2011)

11. P Abel, A Plaza, Clusters versus GPUs for parallel target and anomaly detection in hyperspectral images. EURASIP J Adv Signal Process 2010 915639 (2010)

12. R Xu, DC Wunsch II, Clustering (IEEE/Wiley Press, Hoboken, NJ, 2008)

13. D Everitt, S Landau, M Leese, Clustering Analysis, 4th edn. (Arnold, London, UK, 2001)

14. DC Wunsch, ART properties of interest in engineering applications. Paper presented at the IEEE/INNS international joint conference on neural networks (Atlanta, GA, June 2009), pp. 14-19
15. D Knuth, The Art of Computing Programming: Fundamental Algorithms, vol. 1, 3rd edn. (Addison-Wesley, Boston, 1997)

16. M Martnez-Zarzuela, F Pernas, A de Pablos, M Rodrguez, J Higuera, D Giralda, D Ortega, Adaptative resonance theory fuzzy networks parallel computation using CUDA, bio-inspired systems. Comput Ambient Intell 5517, 149-156 (2009)

17. M Gorchetchnikov, H Ames, M Versace, Simulating Biologically Realistic Neural Models on Graphics Process Units (ICCNS, Boston, 2008)

18. RJ Meuth, GPUs surpass computers at repetitive calculations. Potentials IEEE 26(6), 12-23 (2007)

19. AJ Martin, SM Burns, TK Lee, D Borkovic, PJ Hazewindus, The Design of an Asynchronous Microprocessor (MIT Press, Cambridge, MA, 1986)

20. S Kim, DC Wunsch, A GPU based parallel hierarchical fuzzy ART clustering. Advanced Res 1, 2778-2782 (2003)

21. LI Timchenko, NI Kokryatskaya, VA Melnikov, Method of organization of a parallel-hierarchical network for image recognition. J Cybern Syst Anal 47(1), 140-151 (2011)

22. AA Yarovyy, RS Vlasiuk, Intelligent System of Compression and Recognition of Images Designed for Laser Beam Profiling, vol. 2 (Vinnytsia National Technical University, Ukraine, 2010). pp. 1-11

23. AA Yarovyy, RS Vlasiuk, Simulation Modeling of Neuronetworking Recognition System of Multicolour Spotted Images of Laser Beam Profile, vol. 3 (Vinnytsia National Technical University, Ukraine, 2010). pp. 1-6

24. SU Jung, YS Chung, JH Yoo, KI Chung, U.S. Patent 007697734B2, Method and apparatus of detecting eye using symmetry and moment characteristics of object. 13 April 2010

25. SX Liao, Image analysis by moments (University of Manitoba Winnipeg, Thesis, 1993)

26. RJ Prokop, AP Reeves, A survey of moment-based techniques for unoccluded object representation and recognition. J Graph Model Image Process 54(5), 438-460 (1992)

27. CH Teh, RT Chin, On image analysis by the methods of moments. J IEEE Trans Pattern Anal Machine Intell 10, 496-512 (1988)

28. S Wasson, ATI's Radeon X1000 series GPUs: The graphics game changes again. Tech Report, October 5, 2005. http://techreport.com/review/8864/ ati-radeon-x1000-series-gpus. Accessed 16 April 2013

doi:10.1186/1687-6180-2013-87

Cite this article as: Timchenko et al: Modeling of a method of parallel hierarchical transformation for fast recognition of dynamic images. EURASIP Journal on Advances in Signal Processing 2013 2013:87.

\section{Submit your manuscript to a SpringerOpen ${ }^{\odot}$ journal and benefit from:}

- Convenient online submission

- Rigorous peer review

- Immediate publication on acceptance

- Open access: articles freely available online

- High visibility within the field

- Retaining the copyright to your article

Submit your next manuscript at $>$ springeropen.com 